www.nature.com/aja

\title{
EDITORIAL
}

\section{Clinical evaluation of the infertile male: new options, new challenges}

Asian Journal of Andrology (2012) 14, 3-5; doi:10.1038/aja.2011.73; published online 19 December 2011

$\mathrm{M}$ ale reproductive dysfunction is the sole or contributory cause of infertility in half of couples making the systematic clinical and laboratory evaluation of the male, and the application of costeffective management strategies tailored to the individual patient's need, vital parts of fertility practice. ${ }^{1,2}$ Male infertility has a wide range of etiologies and effective approaches to initiate, restore or preserve natural fertility are available in some settings. But the most striking development in the past 20 years has been in the area of assisted reproductive technologies (ART), especially intracytoplasmic sperm injection (ICSI); the latter provides an extraordinarily effective bypass of the natural processes involved such that a single viable sperm, obtained from any part of the reproductive tract, will often successfully fertilize an oocyte and has allowed many previously sterile men to father healthy children. However, our understanding of the genetic and environmental factors causing male factor infertility has lagged behind these technological advances and still for a significant minority, no options exist other than adoption, donor sperm or abandonment of their aspirations for a family.

It is important that these powerful ART approaches are not allowed to 'corrupt' good clinical practice by inducing: (i) a state of 'diagnostic nihilism' wherein effective, cheaper and less invasive alternative approaches are overlooked; and (ii) a mindset that male reproductive health can be defined by the availability of motile spermatozoa, thereby relieving the clinician of his/her obligation to evaluate and care for infertile men. For example, natural fertility may be restorable and even when specific treatment is not possible, providing the man with a reason for his infertility will assist him in coming to terms with his disability. Furthermore, some health issues are more prevalent in infertile men and must be actively sought (e.g., androgen or gonadotropin deficiency, testicular cancer) and the opportunity taken to assess and improve general and reproductive health. Finally, genetic causes for male infertility must be considered as they may have profound implications for the success of ART and for the health of offspring; in this regard, a close relationship with clinical geneticists is now an essential part of modern ART practice.

All these biomedical considerations must be seen within the patients' psychological and cultural context and the applicable health-care environment. Rigid prescriptive approaches cannot often be made (there may be more than one 'correct answer'), but evidencebased guidelines assist the clinician in making his/her recommendations. A team approach to male infertility, as with broader fertile practice, involves the coordinated efforts of clinicians (andrology, urology, gynecology, endocrinology and primary care), scientific, laboratory, nursing and counseling staff.
In broadly considering the clinical aspects of male infertility, we seek to emphasize a practical approach to evaluation and management, and also to provide the essential background on the pathophysiology of male infertility and emerging research that will translate into practice. At the laboratory interface, semen testing remains the cornerstone of evaluation and the World Health Organization guidelines have been recently revised ${ }^{3}$ and been the subject of recent extensive Reviews in a Special Edition 2010 of Asian Journal of Anrology (http://www. asiaandro.com/Current_Issue_v12_1.asp); ${ }^{4,5}$ implementation of these procedures in different regions is particularly challenging. ${ }^{6}$ Associations between sperm morphology and motility, egg interaction and reproductive outcome are evident but in the clinic, in vitro sperm function testing has not become widespread. ${ }^{6}$ The elaborate structure of sperm is best appreciated at the ultrastructural level; a wide variety of structural defects impact on motility, fertilization and embryonic developmental failure and their proper evaluation informs clinical decision making. ${ }^{7}$ Furthermore, disorders such as primary cilial dyskinesia, can present with a respiratory phenoptype (e.g., Kartageners syndrome), underscoring the need for full clinical evaluation.

Sperm DNA is susceptible to damage, especially from reactive oxygen species; levels of sperm DNA damage levels rise as conventional sperm parameters decline, but are also evident in some men with normal parameters. Consistently high levels of sperm DNA damage is associated with poorer embryonic development, higher pregnancy loss and potentially adverse health effects in offspring. ${ }^{8}$ This area is complicated by the diversity of sperm DNA assessment methods, and controversy exists as to whether sperm DNA measures effectively inform or modify routine clinical practice, ${ }^{9,10}$ or are better directed to the evaluation of couples experiencing repetitive reproductive failure.

Primary spermatogenic failure is a collective term for a heterogenous group of disorders featuring abnormal sperm number, motility, structure and/or and function. It represents by far the largest cohort of infertile men, indeed affecting $\sim 5 \%$ of the general population. While damage from cancer treatments, vascular insult or trauma is readily understood, it is frustrating to both clinicians and patients that most cases are unexplained (idiopathic). Increasingly genetic factors are recognized and their identification is essential in informing couples about the prospect for normal pregnancy, transmission of infertility and/or non-gonadal disease in offspring. ${ }^{11}$ Karyotypic anomalies (numerical, autosomal translocations/inversions) are the commonest recognized cause and result in sterility or degrees of subfertility. ${ }^{12}$ The prevalence of abnormalities is high; $\sim 7 \%$ of men with spermatogenic failure and $\sim 14 \%$ of those with azoospermia, most of the latter having Klinefelter's syndrome. An increased prevalence of aneuploidy in the 
ejaculated or testicular sperm of severely infertile men with a normal peripheral blood karyotype is also reported and may account for higher rate of de novo abnormalities in ICSI offspring. ${ }^{13}$ As most men with abnormal karyotypes are otherwise apparently healthy, routine karyotyping for severely infertile men before ICSI is justified. Clinicians must be informed about the implications of a diagnosis to ART outcome and to the health of offspring in order to properly counsel couples. $^{12}$

Specific genetic defects, such as Y-chromosome microdeletions and CFTR gene mutations, need special consideration. ${ }^{14}$ Yq chromosomal deletions are found in $\sim 5 \%$ of men with severe 'idiopathic' infertility, and testing is now routine prior to ART in men with sperm densities $<5$ million $\mathrm{ml}^{-1}$. Extensive Yq deletions carry a poor prognosis for sperm recovery by testicular sperm extraction. Vertical transmission via ICSI is inevitable and will be associated with spermatogenic defects in these boys. Great care must be taken in performing this PCR-based testing so as to avoid false detection; external quality assurance is essential. ${ }^{15}$ According to four meta-analyses, a partial AZF $c$ deletion named the gr/gr deletion appears to be a significant risk factor for infertility, but the clinical significance of this variant has been questioned in some populations. ${ }^{16}$

Despite extensive research into the genetics of male infertility, relatively few genetic tests are available in the clinic, especially when considering the predicted number of genetic defects. However, the availability of novel whole genome approaches encourages future studies, and will likely contribute to the identification of novel, recurrent genetic factors.

Hypogonadotropic hypogonadism is an uncommon but important cause of infertility that must be determined by clinical laboratory evaluation and imaging. The field of the genetic regulation of puberty has progressed dramatically with the identification of the integrated network of genes and proteins that orchestrate normal puberty, thereby providing new insights into the pathophysiology of delayed/ absent puberty and infertility. ${ }^{17}$ The screening for candidate gene mutations is feasible in most of the routine genetic laboratories and mutation(s) can be identified in about $40 \%$ of congenital forms. Since these patients are largely responsive to medical therapy, the genetic screening has not only a diagnostic value, but also relevance for an appropriate genetic counseling.

Thorough evaluation may reveal medical treatment options to restore fertility and avoid the need for expensive ART options. ${ }^{18}$ Natural fertility may be restored through withdrawal of spermatogenic toxins, cessation of anabolic steroid usage, surgery in suitable obstructive cases or management of erectile or ejaculatory dysfunction. In hypogonadotropic hypogonadism due to hypothalamopituitary disease, treatment with human chorionic gonadotropin, as a luteinizing hormone substitute, restores intratesticular testosterone levels and spermatogenesis in many postpubertal onset cases, but optimal sperm output often requires the addition of follicle-stimulating hormone, and the latter combination is always needed in prepubertal onset cases. If poor sperm output remains so poor as to preclude natural fertility, in vitro fertilization options are readily employed. Background natural fertility rates in subfertile men requires evidence be obtained from placebo-controlled randomized clinical trials for any proposed intervention, and that the clinician must look to see the data (or lack thereof) behind popularized and commercially promoted empirical treatments, such as empirical vitamin, antibiotic and antioxidant treatments.

Many neurological disorders affect the central processes and peripheral nerve function required for normal sexual function and fertility. ${ }^{19}$ In some cases, erectile dysfunction is a coexistent concern and in some may is the explanation for infertility. In other settings, libido and erectile function may be normal, but the coordination of the ejaculatory process fails. These diverse disorders require systematic evaluation and consideration of the least invasive approaches to treatment.

A trend to older parenthood amongst men and women is clear; while the impact of female age on reproductive fitness is well recognized, the adverse effects of male aging on gamete health, reproductive success and the health of offspring are not. ${ }^{20}$ Similarly, the adverse effects on reproductive hormones, sexual function and fertility of prevalent diseases, such as metabolic syndrome, diabetes and other lifestyle related health issues, also go widely unrecognized. ${ }^{21}$ The association of male infertility with complex systemic disorders, such as obesity, represents an opportunity to improve health conditions that, if left untreated, carry significant morbidity and the need for expensive medical interventions. A holistic health approach to the infertile male may produce both reproductive and long-term health benefit.

The 'azoospermic man' is a classic presentation and the differentiation of spermatogenic failure (non-obstructive) from obstructive azoospermia has major implications for management. A systematic approach based on a clinical algorithm ensures timely and cost-effective diagnosis and treatment. ${ }^{22}$ The distinction is usually evident from clinical parameters (history, testis size, semen volume/pH) and serum folliclestimulating hormone levels, but testis biopsy is needed in some cases. Testicular biopsy ${ }^{23}$ reveals the type and severity of spermatogenic failure and has prognostic significance for surgical sperm recovery for ICSI. It also allows detection of incidental testis cancer, which is more common in infertile men, especially those with a history of cryptorchidism. Imprecise terminology make interpretation of the literature difficult; clinically relevant and systematic reporting should be used, while the flawed was yet still widely used Johnsen score should be abandoned. ${ }^{23,24}$

Surgical approaches to correcting genital tract obstruction and congenital anomalies highlights the important role for surgery as a cost-effective approach to restoring fertility and patient reproductive autonomy. ${ }^{25}$ Clinical recommendations for evaluation and management place of varicocele ligation continues to provoke debate; new data are emerging about the impact of varicocele on spermatogenesis and sperm DNA, and the impact of intervention on fertility.

ART approaches now provide paternity to many infertile and previously sterile men. With improved clinical and embryological approaches, success rates have improved and adverse outcomes, such as multiple births, are declining. Many discussions now occur about the cost effectiveness of ART versus and medical/surgical alternatives, and these must take into account local services, expertise, health system costing, and patient demographic factors and expectations. The overarching concept is that the least invasive and most cost-effective approach should be tailored to the individual patient. ${ }^{26}$ In obstructive azoospermia, the decision to attempt surgical correction, as opposed to sperm retrieval and ICSI, is affected by the underlying anatomy, surgical access and skill, and the presence of female cofactors. Surgical sperm recovery approaches to obstructive or non-obstructive azoospermia are now major activities in ART programs. Remarkably, even in severe spermatogenic failure, including Klinefelter's syndrome, testicular sperm can be isolated in $30 \%-60 \%$ of men via needle aspiration or random open biopsy, but higher recovery rates are reported using microdissection testicular sperm extraction. ${ }^{27}$ Thorough and realistic discussion is required regarding the prospects for live birth, androgen deficiency as a result of testicular damage, potential health issues in offspring and the donor sperm alternative. 
Considerable attention has rightfully been given to the health offspring conceived through ART. In male factor infertility, the added concern stems from that use of suboptimal gametes for conception that may carry the risk of vertical transmission of genetic defects while levels of sperm DNA damage are linked to miscarriage and birth defects. To identify differences and properly inform prospective patients, outcome data for male factor etiologies should be monitored and compared with that of other ART populations. Overall, the data seem fairly reassuring, but further data collection and research is needed. ${ }^{28}$ Finally, it must be remembered that male infertility can profoundly affect the individual and couple health and happiness; it is incumbent on medical staff to anticipate, prevent or ameliorate such distress. ${ }^{29}$

The field of male infertility has seen much progress, but in many cases, the pathophysiology remains obscure, and we are unable to provide a precise diagnosis or therapy to restore natural fertility and thus must resort to ART. A great deal more remains to be done to identify the likely genetic and environmental factors responsible. New diagnostic and therapeutic approaches must be properly evaluated through well-designed clinical studies; this is most important in reproductive medicine where empirical and anecdotal reports seem to take hold readily.

\section{(Guest editors of this issue) Robert I McLachlan ${ }^{1}$} and Csilla Krausz

${ }^{1}$ Prince Henry's Institute of Medical Research, Monash Medical Centre,

Clayton, Victoria 3186, Australia (rob.mclachlan@princehenrys.org) and ${ }^{2}$ Andrology Unit, Department of Clinical Physiopathology, University of Florence, Florence, Italy(c.krausz@dfc.unifi.it)

\section{ACKNOWLEDGMENTS}

We would like to thank all the reviewers for their diligent and valuable assistance in reviewing all articles for this special issue of AJA.

1 Baker HW. Clinical Management of Male Infertility in Male Reproduction, Chap. 7, de Groot L, McLachlan R, editors. South Dartmouth, MA: MDText Com. Inc. 2008.

2 Krausz C. Male infertility: pathogenesis and clinical diagnosis. Best Pract Res Clin Endocrinol Metab 2011; 25: 271-85.

3 World Health Organization. WHO Laboratory Manual for the Examination and Processing of Human Semen, 5th ed. Geneva: World Health Organization; 2010.

4 Handelsman DJ, Cooper TG. Foreword to Semen Analysis in 21st Century Medicine special issue in Asian Journal of Andrology. Asian J Androl 2010; 12: 7-10.

5 Handelsman DJ, Cooper TG. Afterword to Semen Analysis in 21st Century Medicine special issue in Asian Journal of Andrology. Asian J Androl 2010; 12: 118-23.
6 Franken DR, Oehninger S. Semen analysis and sperm function testing. Asian J Androl; 2012; 14: 6-13.

7 Chemes HE, Sedo CA. Tales of the tail and sperm head aches: changing concepts on the prognostic significance of sperm pathologies affecting the head, neck and tail. Asian J Androl 2012; 14: 14-23.

8 Tamburrino L, Marchiani S, Montoya M, Marino FE, Natali I et al. Mechanisms and clinical correlates of sperm DNA damage. Asian J Androl 2012; 14: 24-31.

9 Zini A, Jamal W, Cowan L, Al-Hathal N. Is sperm DNA damage associated with IVF embryo quality? A systematic review. J Assist Reprod Genet 2011; 28: 391-7.

10 Barratt CL, Aitken RJ, Björndahl L, Carrell DT, de Boer P et al. Sperm DNA organization, protection and vulnerability: from basic science to clinical applications-a position report. Hum Reprod 2010; 25: 824-38.

11 McLachlan RI, O'Bryan MK. Clinical review: state of the art for genetic testing of infertile men. J Clin Endocrinol Metab 2010; 95: 1013-24.

12 Harton GL, Tempest HG. Chromosomal disorders and male infertility. Asian J Androl 2012; 14: 32-39.

13 Bonduelle M, van Assche E, Joris H, Keymolen K, Devroey P et al. Prenatal testing in ICSI pregnancies: incidence of chromosomal anomalies in 1586 karyotypes and relation to sperm parameters. Hum Reprod 2002; 17: 2600-14.

14 Massart A, Lissens W, Tournaye H, Stouffs K. Genetic causes of spermatogenic failure. Asian J Androl 2012; 14: 40-48.

15 Simoni M, Bakker E, Krausz C. EAA/EMQN best practice guidelines for molecular diagnosis of Y-chromosomal microdeletions. State of the art 2004. Int J Androl 2004; 27: 240-9.

16 Krausz C, Chianese C, Giachini C, Guarducci E, Laface I et al. The Y chromosomelinked copy number variations and male fertility. J Endocrinol Invest 2011; 34: 376 82

17 Bonomi M, Libri DV, Guizzardi F, Guarducci E, Maiolo E et al. The Idiopathic Central Hypogonadism Study Group of the Italian Societies of Endocrinology and Pediatric Endocrinology and Diabetes. New understandings of the genetic basis of isolated idiopathic central hypogonadism. Asian J Androl 2012; 14: 49-56.

18 Ramasamy R, Stahl PJ, Schlegel PN. Medical therapy for spermatogenic failure. Asian $J$ Androl 2012; 14: 57-60.

19 Fode M, Krogh-Jespersen S, Brackett NL, OhI DA, Lynne CM et al. Male sexua dysfunction and infertility associated with neurological disorders. Asian J Androl 2012; 14: 61-8.

20 Wiener-Megnazi Z, Auslender R, Dirnfeld M. Advanced paternal age and reproductive outcome. Asian J Androl 2012; 14: 69-76.

21 Hammoud AO, Carrell DT, Gibson M, Peterson CM, Meikle AW. Updates on the relation of weight excess and reproductive function in men: sleep apnea as a new area of interest. Asian J Androl 2012; 14: 77-81.

22 Oates R. Evaluation of the azoospermic male. Robert Oates. Asian J Androl2012; 14 82-7.

23 Dohle GR, Elzanaty S, van Casteren NJ. Testicular biopsy: clinical practice and interpretation. Asian J Androl 2012; 14: 88-93.

24 McLachlan RI, Rajpert-De Meyts E, Hoei-Hansen CE, de Kretser DM, Skakkebaek NE. Histological evaluation of the human testis-approaches to optimizing the clinical value of the assessment: mini review. Hum Reprod 2007; 22: 2-16.

25 Lopushnyan NA, Walsh TJ. Surgical techniques for the management of male infertility. Asian J Androl 2012; 14: 94-102.

26 Tournaye H. Male factor infertility and ART. Asian J Androl 2012; 14: 103-8.

27 Ishikawa T. Surgical recovery of sperm in non-obstructive azoospermia. Asian J Androl 2012; 14: 109-15.

28 Halliday J. Outcomes for offspring of men having ICSI for male factor infertility. Asian J Androl 2012; 14: 116-20.

29 Fisher JR, Hammarberg K. Psychological and social aspects of infertility in men: an overview of the evidence and implications for psychologically informed clinical care and future research. Asian J Androl 2012; 14: 121-9. 